\title{
Improvements on the Convergence and Stability of Fuzzy Grey Cognitive Maps
}

\author{
István Á. Harmati ${ }^{1(凶)}$ and László T. Kóczy ${ }^{2,3}$ \\ 1 Department of Mathematics and Computational Sciences, \\ Széchenyi István University, Egyetem tér 1, Györ 9026, Hungary \\ harmati@sze.hu \\ 2 Department of Information Technology, Széchenyi István University, \\ Egyetem tér 1, Győr 9026, Hungary \\ koczy@sze.hu \\ 3 Department of Telecommunication and Media Informatics, \\ Budapest University of Technology and Economics, Magyar tudósok körútja 2, \\ Budapest 1117, Hungary
}

\begin{abstract}
Fuzzy grey cognitive maps (FGCMs) are extensions of fuzzy cognitive maps (FCMs), where the causal connections between the concepts are represented by so-called grey numbers. Just like in classical FCMs, the inference is determined by an iteration process, which may converge to an equilibrium point, but limit cycles or chaotic behaviour may also show up.

In this paper, based on network measures like in-degree, out-degree and connectivity, we provide new sufficient conditions for the existence and uniqueness of fixed points for FGCMs. Moreover, a tighter convergence condition is presented using the spectral radius of the modified weight matrix.
\end{abstract}

Keywords: Fuzzy cognitive map - Fuzzy grey cognitive map • Stability $\cdot$ Convergence $\cdot$ Equilibrium point

\section{Introduction}

Fuzzy cognitive maps are neural network-based decision support tools, where the neurons represent specific factors or characteristics of the modelled system [11]. Graphically, a fuzzy cognitive map is a weighted, directed graph. The constant weights assigned to the edges from the interval $[-1,1]$ express the strength and direction of causal connections. The current states of the neurons (which are called concepts in FCM literature) are also characterized by numbers in the $[0,1]$ interval (in some applications the interval $[-1,1]$ is also applicable [12]). These are the activation values of the concepts [6]. 
Formally, the system can be described by the set of concepts $\left(C_{1}, C_{2}, \ldots, C_{n}\right)$; the current activation values of the concepts $\left(A_{1}, A_{2}, \ldots, A_{n}\right)$; the weight matrix $W$ which assigns weight $w_{i j}$ to each edge connecting the nodes $C_{i}$ and $C_{j}$ ), expressing how strongly influenced is concept $C_{i}$ by concept $C_{j}$. The sign of $w_{i j}$ indicates whether the relationship between $C_{j}$ and $C_{i}$ is direct or inverse. So matrix $W$ represents the weighted causal connections between the concepts. A transformation function $f: \mathbb{R} \rightarrow[0,1]$ calculates the activation value of concepts at every time step of the iteration and the activation values in the allowed range (sometimes a function $f: \mathbb{R} \rightarrow[-1,1]$ is applied).

The iteration rule which calculates the values of the concept at every step may or may not include self-feedback. In general form it can be written as

$$
A_{i}(k)=f\left(\sum_{j=1, j \neq i}^{n} w_{i j} A_{j}(k-1)+d_{i} A_{i}(k-1)\right)
$$

where $A_{i}(k)$ is the value of concept $C_{i}$ at discrete time $k, w_{i j}$ is the weight of the connection from concept $C_{j}$ to concept $C_{i}$ and $0 \leq d_{i} \leq 1$ expresses the possible self-feedback. If $d_{i}=0$, then there is no self-feedback. If we include the $d_{i}$ s into the diagonal of weight matrix $W$, the iteration equation can be rewritten in more compact style:

$$
A_{i}(k+1)=f\left(\sum_{j=1}^{n} w_{i j} A_{j}(k)\right)=f\left(w_{i} A(k)\right),
$$

where $w_{i}=\left[w_{i 1}, \ldots, w_{i n}\right]$ is the $i$ th row of $W$ and $A(k)=\left[A_{1}(k), \ldots, A_{n}(k)\right]^{T}$ is the concept vector after $k$ iterations. We apply dot product between them, so $w_{i} A^{(k)}$ is a real number.

Moreover, if we couple the coordinates of the concept vector together and denote by $G$ the mapping $\mathbb{R}^{n} \rightarrow \mathbb{R}^{n}$ that generates the concept vector $A(k+1)$ from $A(k)$, then we have that:

$$
A(k+1)=\left[\begin{array}{c}
A_{1}(k+1) \\
\vdots \\
A_{n}(k+1)
\end{array}\right]=\left[\begin{array}{c}
f\left(w_{1} A(k)\right) \\
\vdots \\
f\left(w_{n} A(k)\right)
\end{array}\right]=G(A(k)) .
$$

The iteration rule repeated until either the FCM converges to an equilibrium state (fixed point) or the maximal number of iterations is reached. Mathematically, the FCM may converge to a fixed point, may arrive to a limit cycle or shows chaotic pattern $[2,5]$.

The weights of the connections are usually determined by human experts or by learning methods. In both of the cases there are some uncertainties about the exact values of the weights. This was the main motivation of Fuzzy Grey Cognitive Maps, where the weights and concept values are modelled by the socalled grey numbers $[8-10,13]$. 
A grey number (denoted by $\otimes g$ ) is a number whose accurate value is unknown, but we know the range within the value is included. A grey number with both a lower limit $(g)$ and an upper limit $(\bar{g})$ is called an interval grey number [4], so $\otimes g \in[g, \bar{g}]$. In applications, a grey number is usually an interval. The basic arithmetic operations on grey numbers are the following [4]:

1. $\otimes g_{1}+\otimes g_{2} \in\left[\underline{g_{1}}+\underline{g_{2}}, \overline{g_{1}}+\overline{g_{2}}\right]$

2. $-\otimes g \in[-\bar{g},-\underline{-g}]$

3. $\otimes g_{1}-\otimes g_{2} \in\left[\underline{g_{1}}-\overline{g_{2}}, \overline{g_{1}}-g_{2}\right]$

4. $\otimes g_{1} \times \otimes g_{2} \in[\overline{\min }(S), \max \overline{(S)}]$,

where $S=\left\{\underline{g_{1}} \cdot \underline{g_{2}}, \underline{g_{1}} \cdot \overline{g_{2}}, \overline{g_{1}} \cdot \underline{g_{2}}, \overline{g_{1}} \cdot \overline{g_{2}}\right\}$

5. If $\lambda>0, \lambda \in \overline{\mathbb{R}}$, then $\lambda \cdot \otimes g \in \overline{[\lambda} g, \lambda \bar{g}]$

Beside the above defined operations, we have to provide a consistent definition for the generalization of any $f: \mathbb{R} \rightarrow \mathbb{R}$ function to grey numbers. The function of a grey number $\otimes g \in[\underline{g}, \bar{g}]$ is the grey number $f(\otimes g) \in[\underline{f(\otimes g)}, \overline{f(\otimes g)}]$, where

$$
\begin{aligned}
& \underline{f(\otimes g)}=\inf \{f(\gamma): \gamma \in[\underline{g}, \bar{g}]\} \\
& \overline{f(\otimes g)}=\sup \{f(\gamma): \gamma \in[\underline{g}, \bar{g}]\}
\end{aligned}
$$

For a continuous and monotone increasing function $f$ we have

$$
\begin{aligned}
\inf \{f(\gamma): \gamma \in[\underline{g}, \bar{g}]\} & =f(\underline{g}) \\
\sup \{f(\gamma): \gamma \in[\underline{g}, \bar{g}]\} & =f(\bar{g})
\end{aligned}
$$

Consequently, $f(\otimes g)=f(\underline{g})$ and $\overline{f(\otimes g)}=f(\bar{g})$ and $f(\otimes g) \in[f(\underline{g}), f(\bar{g})]$.

The dynamics of an FGCM is similar to the original FCM's. It begins with an initial grey vector $A(0)$, which represents initial uncertainty. The elements of this vector are grey numbers, i.e. $A_{i}(0) \in\left[A_{i}(0), \overline{A_{i}(0)}\right]$ for every $i$. The activation values are computed by the iterative process, resulting grey numbers as concept values:

$$
A_{i}(k) \in\left[f\left(\underline{w_{i} A(k-1)}\right), f\left(\overline{w_{i} A(k-1)}\right)\right]
$$

An FGCM with continuous threshold produces one of the following behaviours:

1. Fixed point: the FGCM converges to a grey fixed-point attractor. This fixed point is vector, whose coordinates are grey numbers (intervals). The convergence (stabilization) means that the endpoints of these intervals are stabilized after a certain number of iterations.

2. Limit cycle: the state values keep oscillating between several states. These states (elements of the limit cycle) are concept vectors with interval coordinates.

3. Chaotic behaviour: the FGCM produces different grey vector states for each iteration, without any pattern. 
Usually, the behaviour of fuzzy cognitive maps is examined by trial-error methods. The main contribution of this paper is to present analytical conditions for the existence and uniqueness of attracting fixed points of FGCMs. It also ensures the global exponential stability of the system. Previously, Boutalis et al. [14] proved a condition for the convergence of a class of FCMs. Their result has been generalized in [2]. Knight et al. [15] studied the problem of fixed points of FCMs using only the topology, without the weights.

In this paper, we give several conditions for convergence and stability of fuzzy grey cognitive maps. In Sect. 2 different type of behaviours of FCMs and FGCMs are demonstrated by illustrative examples. In Sect. 3 we briefly summarize the mathematical background, in Sect. 4 some theorems are proved regarding to existence and uniqueness of fixed points of FGCMs. We illustrate the results with an example in Sect. 5, and shortly summarize them in Sect. 6.

\section{Examples for Different Behaviour}

Consider the following toy example to demonstrate the behaviour of FCMs and FGCMs (Fig. 1). Although this network is extremely simple, it is able to produce qualitatively different behaviours for different choice of weights. Let us apply the hyperbolic tangent function with parameter $\lambda(\tanh (\lambda x))$ as threshold function (for some properties of hyperbolic tangent FCMs see [3]).

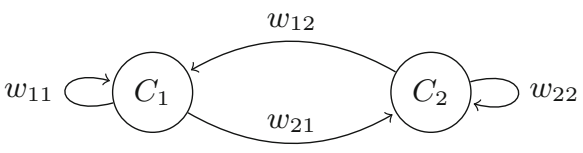

Fig. 1. The topology of the demonstrative example. The self-loops indicate the possible existence of self-feedback.

Different settings of weights and parameter $\lambda$ yield completely different behaviour, although the topology remains the same.

For a certain set of parameters we may have a non-trivial fixed point (the trivial fixed point is the zero vector, since it is always a fixed point of hyperbolic tangent FCMs, but not always attractor [3]) (Fig. 2). Other setting yields oscillation, namely a quasiperiodic behaviour (Fig. 3).

Convergence of FGCMs means that the upper and lower endpoints of the intervals containing the activations values are stabilized. It can be observed in Fig. 4, while with different weights and parameter $\lambda$ we can observe oscillating pattern (Fig. 5). 


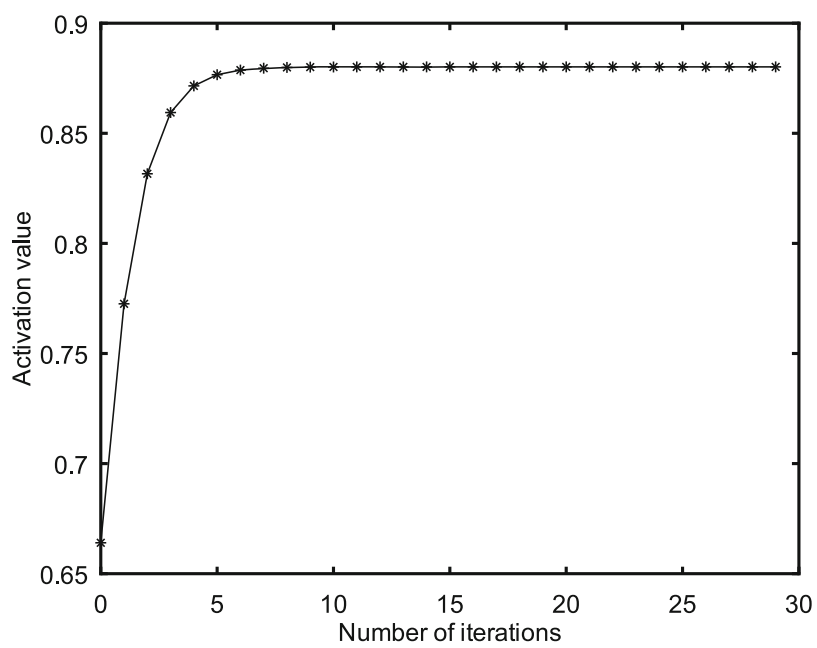

Fig. 2. FCM with hyperbolic tangent threshold function: fixed point. The activation value of concept $C_{1}$ vs. number of iterations. The parameters are $w_{11}=1, w_{21}=$ $0.6, w_{21}=0.4, w_{22}=1, \lambda=1$.

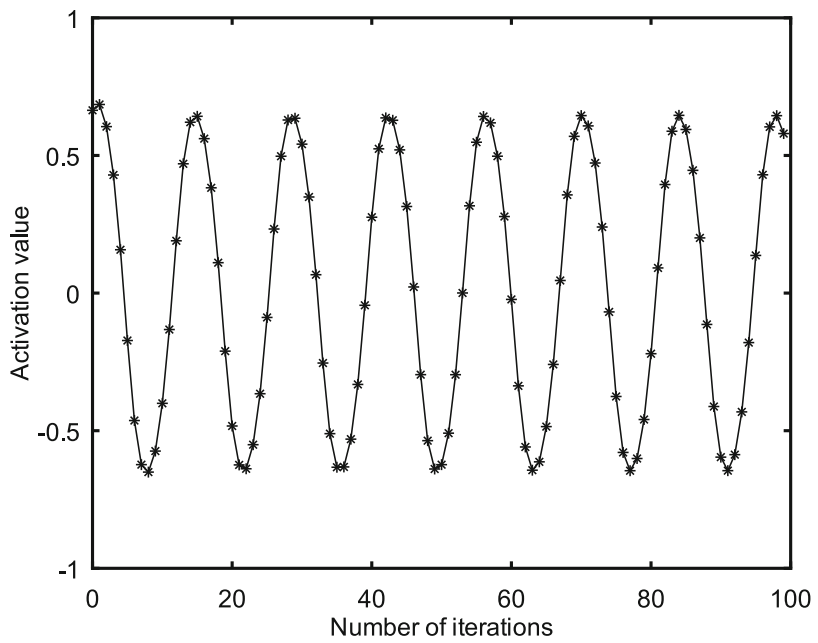

Fig. 3. FCM with hyperbolic tangent threshold function: quasiperiodic pattern. The activation value of concept $C_{1}$ vs. number of iterations. The parameters are $w_{11}=$ $1, w_{21}=0.6, w_{21}=-0.4, w_{22}=1, \lambda=1$. 


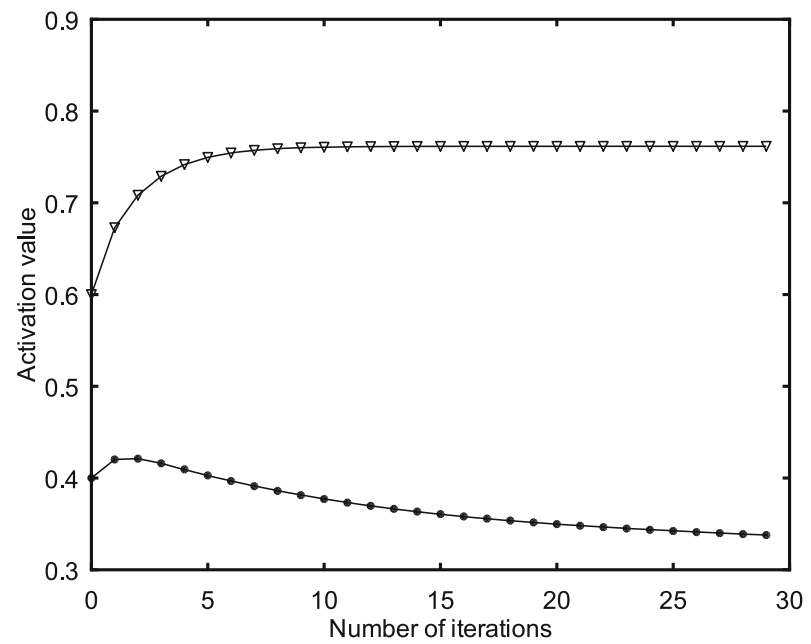

Fig. 4. FGCM with hyperbolic tangent threshold function: fixed point. The activation value (interval) of concept $C_{1}$ vs. number of iterations. The upper endpoint of the interval is denoted by $\nabla$, the lower endpoint is denoted by $\bullet$. The parameters are $w_{11}=[0.9,1], w_{21}=[0.5,0.7], w_{21}=[0.3,0.5], w_{22}=[0.9,1], \lambda=0.8$.

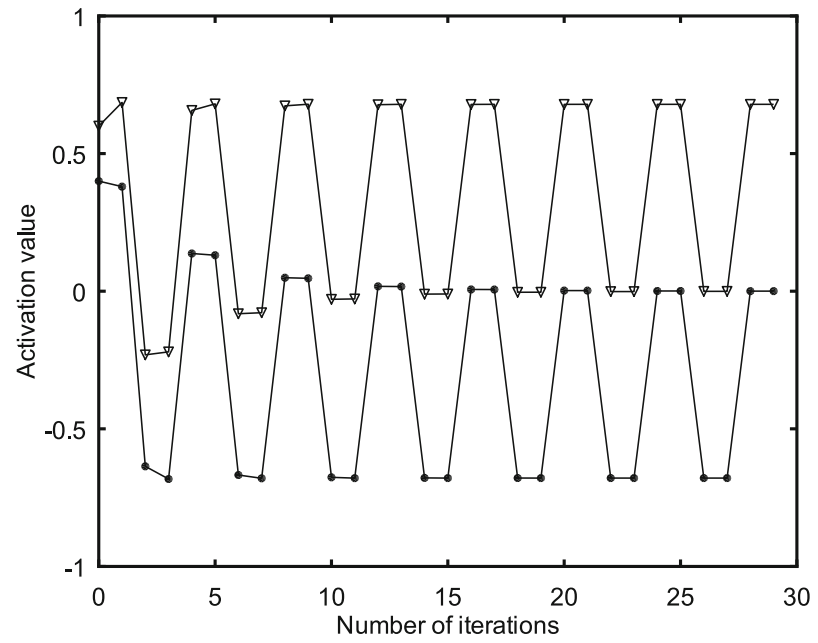

Fig. 5. FGCM with hyperbolic tangent threshold function: oscillating behaviour. The activation value (interval) of concept $C_{1}$ vs. number of iterations. The upper endpoint of the interval is denoted by $\nabla$, the lower endpoint is denoted by $\bullet$. The parameters are $w_{11}=0, w_{21}=[0.5,0.7], w_{21}=[-0.5,-0.3], w_{22}=0, \lambda=2$. 


\section{Mathematical Background}

The results presented in the next section are based on the contraction property of the mapping that generates the iteration. Here we recall the definition of contraction mapping [7]:

Definition 1. Let $(X, d)$ be a metric space. A mapping $G: X \rightarrow X$ is a contraction mapping or contraction if there exists a constant $c$ (independent from $x$ and $y)$, with $0 \leq c<1$, such that

$$
d(G(x), G(y)) \leq c d(x, y)
$$

The notion of contraction is related to the distance metric $d$ applied. It may happen that a function is a contraction w.r.t. one distance metric, but not a contraction w.r.t. another distance metric. The iterative process of an FCM may end at an equilibrium point, which is a so-called fixed point.

Let $G: X \rightarrow X$, then a point $x^{*} \in X$ such that $G\left(x^{*}\right)=x^{*}$ is a fixed point of $G$. The following theorem provides sufficient condition for the existence and uniqueness of a fixed point [7]. Moreover, if mapping that generates the iteration is a contraction, it ensures the stability of the iteration.

Theorem 1 (Banach's fixed point theorem). If $G: X \rightarrow X$ is a contraction mapping on a nonempty complete metric space $(X, d)$, then $G$ has only one fixed point $x^{*}$. Moreover, $x^{*}$ can be found as follows: start with an arbitrary $x_{0} \in X$ and define the sequence $x_{n+1}=G\left(x_{n}\right)$, then $\lim _{n \rightarrow \infty} x_{n}=x^{*}$.

Definition 2. Let $x^{*}$ be a fixed point of the iteration $x_{n+1}=G\left(x_{n}\right)$. $x^{*}$ is locally asymptotically stable if there exist a neighborhood $U$ of $x^{*}$, such that for each starting value $x_{0} \in U$ we get that

$$
\lim _{n \rightarrow \infty} x_{n}=x^{*}
$$

If this neighborhood $U$ is the entire domain of $G$, then $x^{*}$ is a globally asymptotically stable fixed point.

Corollary 1. If $G: X \rightarrow X$ is a contraction mapping on a nonempty complete metric space $(X, d)$, then its unique fixed point $x^{*}$ is globally asymptotically stable.

In Sect. 4, the following property of the sigmoid function will be applied:

The derivative of the sigmoid function $f: \mathbb{R} \rightarrow \mathbb{R}, f(x)=1 /\left(1+e^{-\lambda x}\right)$, $(\lambda>0)$ is bounded by $\lambda / 4$. Moreover, for every $x, y \in \mathbb{R}$ the following inequality holds

$$
|f(x)-f(y)| \leq \lambda / 4 \cdot|x-y|
$$

In [1] the following statements have been introduced about the convergence of fuzzy grey cognitive maps: 
Theorem 2. Let $\otimes W$ be the extended (including possible feedback) weight matrix of a fuzzy grey cognitive map (FGCM), where the weights $\otimes w_{i j}$ are nonnegative or nonpositive grey numbers and let $\lambda>0$ be the parameter of the sigmoid function $f(x)=1 /\left(1+e^{-\lambda x}\right)$ applied for the iteration. Let $W^{*}$ be a matrix defined by the absolute values of the weights, i.e. $w_{i j}^{*}=\max \left\{\left|\underline{w_{i j}}\right|,\left|\overline{w_{i j}}\right|\right\}$. If one of the inequalities

$$
\begin{aligned}
\left\|W^{*}\right\|_{1} & <\frac{4}{\lambda} \\
\left\|W^{*}\right\|_{\infty} & <\frac{4}{\lambda} \\
\left\|W^{*}\right\|_{F} & <\frac{4}{\lambda}
\end{aligned}
$$

hold, then the FGCM has one and only one grey fixed point, regardless of the initial concept values.

Here $\|*\|_{1},\|*\|_{\infty}$ and $\|*\|_{F}$ denote the 1-norm, infinity norm and Frobenius norm of the matrix, respectively. Here fixed point $\otimes A^{*}$ is

$$
\otimes A^{*}=\left[\otimes A_{1}^{*}, \ldots, \otimes A_{n}^{*}\right]^{T} \in\left[\left[\underline{A_{1}^{*}}, \overline{A_{1}^{*}}\right], \ldots,\left[\underline{A_{n}^{*}}, \overline{A_{n}^{*}}\right]\right]^{T}
$$

The grey fixed point is unique in the sense that the endpoints of the intervals containing grey concept values are unique, i.e. the values $\underline{A_{i}^{*}}$ and $\overline{A_{i}^{*}}$ are unique for every $i$.

\section{Convergence Conditions}

In this section, we provide several theorems regarding the existence and uniqueness of attracting grey fixed point. The first three theorems are based on the structure of the FGCM, namely they are based in the so-called in-degree, outdegree and connectivity, which are widely used measures to describe the quality of the network. The last one is based on the spectral radius of the modified weight matrix $W^{*}$ and it gives the better condition in the sense that it ensures the convergence for the largest set of parameter $\lambda$.

Definition 3. The weighted in-degree of concept $C_{j}$ equals the sum of the absolute values of the weights of in-coming edges:

$$
\operatorname{deg}_{j}^{i n}=\sum_{i=1}^{n}\left|w_{i j}\right|
$$

which is the sum of the absolute values of the entries of the $j$ th column of $W$. 
Definition 4. The weighted out-degree of concept $C_{i}$ equals the sum of the absolute values of the weights of out-going edges:

$$
\operatorname{deg}_{i}^{\text {out }}=\sum_{j=1}^{n}\left|w_{i j}\right|
$$

which is the sum of the absolute values of the entries of the ith row of $W$.

We note that self-feedback means self-loop in the graph. So if self-feedbacks are applied in the concepts, then the weights of the feedback are counted in the in-degree and the out-degree, too. It is the reason that we did not exclude $i=j$ from the summations above.

Definition 5. The connectivity of an FCM is the ratio of the number of connections between concepts to the maximum number of such possible connections.

Connectivity measures the 'density' of the network. If self-feedback is allowed, then the maximum number of connections is $n^{2}$, if not allowed, then the maximum number of connections is $n(n-1)$.

The weighted in-degree, weighted out-degree and weighted connectivity can defined similarly for FGCMs, but instead of absolute values of real numbers (exact weights), we use the absolute values of grey numbers (intervals):

$$
\begin{aligned}
\operatorname{deg}_{j}^{i n} & =\sum_{i=1}^{n}\left|\otimes w_{i j}\right|=\sum_{i=1}^{n} w_{i j}^{*} \\
d e g_{i}^{\text {out }} & =\sum_{j=1}^{n}\left|\otimes w_{i j}\right|=\sum_{j=1}^{n} w_{i j}^{*}
\end{aligned}
$$

Definition 6. The weighted connectivity of an FCM is the ratio of the sum of absolute values of weights of connections between concepts to the maximum number of such possible connections.

If self-feedback is allowed, then the weighted connectivity is

$$
\operatorname{Con}_{w}=\frac{\sum_{i=1}^{n} \sum_{j=1}^{n}\left|w_{i j}\right|}{n^{2}}
$$

If self-feedback is not allowed, then the weighted connectivity is

$$
\operatorname{Con}_{w}=\frac{\sum_{i=1}^{n} \sum_{j=1}^{n}\left|w_{i j}\right|}{n(n-1)}
$$

For fuzzy grey cognitive maps, we apply the absolute values of the grey weights $\left(w_{i j}^{*}-\mathrm{s}\right)$, so the enumerator is the sum $\sum_{i=1}^{n} \sum_{j=1}^{n} w_{i j}^{*}$.

Theorem 3. Let $\lambda$ be the parameter of the sigmoid threshold function applied for every concept. If the maximal in-degree of the FGCM (including possible feedback) is less than $4 / \lambda$, then the FGCM has one and only one fixed point. 
Proof. In [1] it has been shown that if $\left\|W^{*}\right\|_{1}<4 / \lambda$, then the FGCM has one and only one grey fixed point. Moreover, since

$$
\left\|W^{*}\right\|_{1}=\max _{1 \leq j \leq n} \sum_{i=1}^{n} w_{i j}^{*}=\max _{1 \leq j \leq n} d e g_{j}^{i n}
$$

this condition is equivalent to the requirement stated in the theorem.

Theorem 4. Let $\lambda$ be the parameter of the sigmoid threshold function applied for every concept. If the maximal out-degree of the FGCM (including possible feedback) is less than $4 / \lambda$, then the FGCM has one and only one fixed point.

Proof. The proof goes similarly to the previous one, but instead of 1-norm we use the infinity norm. In [1] it has been shown that if $\left\|W^{*}\right\|_{\infty}<4 / \lambda$, then the FGCM has one and only one grey fixed point. Moreover, since

$$
\left\|W^{*}\right\|_{\infty}=\max _{1 \leq i \leq n} \sum_{j=1}^{n} w_{i j}^{*}=\max _{1 \leq i \leq n} \operatorname{deg}_{i}^{\text {out }}
$$

this condition is equivalent to the requirement stated in the theorem.

The theorems above are mathematically equivalent with the statements of Theorem 2, but they are easier to capture by the users of FCMs. While the users are not necessarily familiar with matrix norms, they can easily handle notions like in- and out-degree, which are graphically straightforward.

Theorem 5. Let $\lambda$ be the parameter of the sigmoid threshold function applied for every concept. If the weighted connectivity $\left(\mathrm{Con}_{w}\right)$ of the FGCM small enough, namely

1. if self-feedback is allowed:

$$
\operatorname{Con}_{w}<\frac{4}{\lambda n^{2}}
$$

2. if self-feedback is not allowed:

$$
\operatorname{Con}_{w}<\frac{4}{\lambda n(n-1)},
$$

then the FGCM has one and only one fixed point.

Proof. We show that if $\sum_{i=1}^{n} \sum_{j=1}^{n} w_{i j}^{*}<4 / \lambda$, then mapping $G$ is a contraction, so it has exactly one fixed point. Let us define the distance of grey concept vectors as

$$
d\left(A, A^{\prime}\right)=\frac{1}{2}\left(\left\|\underline{A}-\underline{A}^{\prime}\right\|_{1}+\left\|\bar{A}-\bar{A}^{\prime}\right\|_{1}\right)
$$

We are going to show that with the distance measure above:

$$
d\left(G(A), G\left(A^{\prime}\right)\right) \leq \frac{\lambda}{4} \sum_{i=1}^{n} \sum_{j=1}^{n} w_{i j}^{*} d\left(A, A^{\prime}\right)
$$


By the definition of the distance of two grey-valued vectors, we have

$$
d\left(G(A), G\left(A^{\prime}\right)\right)=\frac{1}{2}\left(\left\|\underline{G(A)}-\underline{G\left(A^{\prime}\right)}\right\|_{1}+\left\|\overline{G(A)}-\overline{G\left(A^{\prime}\right)}\right\|_{1}\right)
$$

It has been shown in [1] that the following upper estimation can be given for the difference of the $i$ th coordinates (similar inequality holds for the difference of the upper endpoints):

$$
\left|\underline{G(A)}_{i}-{\underline{G\left(A^{\prime}\right)}}_{i}\right| \leq\left|\frac{\lambda}{4} w_{i}^{*}\right| \underline{A}-\underline{A^{\prime}}||
$$

where $w_{i}^{*}$ is the $i$ th row of matrix $W^{*}$ and we apply dot product between $w_{i}^{*}$ and $\left|\underline{A}-\underline{A}^{\prime}\right|=\left(\left|A_{1}-A_{1}^{\prime}\right|, \ldots,\left|A_{n}-A_{n}^{\prime}\right|\right)$. Moreover,

$$
w_{i}^{*}\left|\underline{A}-\underline{A^{\prime}}\right| \leq\left\|w_{i}^{*}\right\|_{1} \cdot\left\|\underline{A}-\underline{A^{\prime}}\right\|_{1}
$$

Here $\left\|w_{i}^{*}\right\|_{1}=\sum_{j=1}^{n}\left|w_{i j}^{*}\right|=\sum_{j=1}^{n} w_{i j}^{*}$. Use this inequality for the distance of $G(A)$ and $G\left(A^{\prime}\right)$ :

$$
\begin{aligned}
& d\left(G(A), G\left(A^{\prime}\right)\right)=\frac{1}{2}\left(\left\|\underline{G(A)}-\underline{G\left(A^{\prime}\right)}\right\|_{1}+\left\|\overline{G(A)}-\overline{G\left(A^{\prime}\right)}\right\|_{1}\right) \\
& =\frac{1}{2}\left(\sum_{i=1}^{n}\left|\underline{G(A)}_{i}-{\underline{G\left(A^{\prime}\right)_{i}}}_{i}\right|+\sum_{i=1}^{n}\left|\overline{G(A)}_{i}-\overline{G\left(A^{\prime}\right)_{i}}\right|\right) \\
& \leq \frac{\lambda}{4} \frac{1}{2}\left(\sum_{i=1}^{n} \sum_{j=1}^{n} w_{i j}^{*}\left\|\underline{A}-\underline{A}^{\prime}\right\|_{1}+\sum_{i=1}^{n} \sum_{j=1}^{n} w_{i j}^{*}\left\|\bar{A}-\bar{A}^{\prime}\right\|_{1}\right) \\
& =\frac{\lambda}{4} \sum_{i=1}^{n} \sum_{j=1}^{n} w_{i j}^{*} \frac{1}{2}\left(\left\|\underline{A}-\underline{A^{\prime}}\right\|_{1}+\left\|\bar{A}-\bar{A}^{\prime}\right\|_{1}\right) \\
& =\frac{\lambda}{4} \sum_{i=1}^{n} \sum_{j=1}^{n} w_{i j}^{*} \cdot d\left(A, A^{\prime}\right)
\end{aligned}
$$

If $\frac{\lambda}{4} \sum_{i=1}^{n} \sum_{j=1}^{n} w_{i j}^{*}<1$, then the mapping is a contraction, so the iteration leads to a unique fixed point, regardless to the initial value. Rearanging this inequality and division both sides by $n^{2}($ or $n(n-1))$ completes the proof.

Although Theorem 5 provides weaker condition, it has an important message expressed by connectivity: poorly connected FGCMs cannot produce complex behaviour (the term 'poorly' depends on $\lambda$ and $n$ ).

Theorem 6. Let $\otimes W$ be the extended (including possible feedback) weight matrix of a fuzzy grey cognitive map (FGCM), where the weights $\otimes w_{i j}$ are nonnegative or nonpositive grey numbers and let $\lambda>0$ be the parameter of the sigmoid function $f(x)=1 /\left(1+e^{-\lambda x}\right)$ applied for the iteration. Let $W^{*}$ be a 
matrix defined by the absolute values of the weights. If the spectral radius of $W^{*}$ is less than $4 / \lambda$, i.e if the inequality

$$
\rho\left(W^{*}\right)<\frac{4}{\lambda}
$$

hold, then the FGCM has one and only one grey fixed point, regardless of the initial concept values.

Proof. Let us define the distance of two grey-valued vectors as the norm of their difference. At this stage we do not specify this norm:

$$
d\left(A, A^{\prime}\right)=\left\|A-A^{\prime}\right\|=\frac{1}{2}\left(\left\|\underline{A}-\underline{A}^{\prime}\right\|+\left\|\bar{A}-\bar{A}^{\prime}\right\|\right)
$$

We are going to show that with the distance above and for a suitable matrix norm:

$$
d\left(G(A), G\left(A^{\prime}\right)\right) \leq \frac{\lambda}{4}\left\|W^{*}\right\| d\left(A, A^{\prime}\right)
$$

By the definition of the distance of two grey-valued vectors, we have

$$
d\left(G(A), G\left(A^{\prime}\right)\right)=\frac{1}{2}\left(\left\|\underline{G(A)}-\underline{G\left(A^{\prime}\right)}\right\|+\left\|\overline{G(A)}-\overline{G\left(A^{\prime}\right)}\right\|\right)
$$

It has been shown in [1] that the following upper estimation can be given for the difference of the $i$ th coordinates (similar inequality holds for the difference of the upper endpoints):

$$
\left|\underline{G(A)}_{i}-\underline{G\left(A^{\prime}\right)} i\right| \leq\left|\frac{\lambda}{4} w_{i}^{*}\right| \underline{A}-\underline{A^{\prime}}||
$$

where $w_{i}^{*}$ is the $i$ th row of matrix $W^{*}$. Since this inequality holds for every coordinates, we conclude to following inequality for the difference of the lower endpoint vectors:

$$
\left\|\underline{G(A)}-\underline{G\left(A^{\prime}\right)}\right\| \leq\left\|\frac{\lambda}{4} W^{*}\left|\underline{A}-\underline{A^{\prime}}\right|\right\|
$$

Using this inequality (and the corresponding inequality for the upper endpoints) we provide upper estimation for the distance of $G(A)$ and $G\left(A^{\prime}\right)$ :

$$
\begin{aligned}
d\left(G(A), G\left(A^{\prime}\right)\right) & =\frac{1}{2}\left(\left\|\underline{G(A)}-\underline{G\left(A^{\prime}\right) \|}+\right\| \overline{G(A)}-\overline{G\left(A^{\prime}\right)} \|\right) \\
& \leq \frac{1}{2}\left(\left\|\frac{\lambda}{4} W^{*}\left|\underline{A}-\underline{A^{\prime}}\right|\right\|+\left\|\frac{\lambda}{4} W^{*}\left|\bar{A}-\bar{A}^{\prime}\right|\right\|\right) \\
& \leq \frac{\lambda}{4}\left\|W^{*}\right\| \frac{1}{2}\left(\left\|\underline{A}-\underline{A^{\prime}}\right\|+\left\|\bar{A}-\bar{A}^{\prime}\right\|\right) \\
& =\frac{\lambda}{4}\left\|W^{*}\right\| d\left(A, A^{\prime}\right)
\end{aligned}
$$

In $\left\|W^{*}\right\|$, the matrix norm is induced by the vector norm. By the contraction mapping theorem, if the coefficient of $d\left(A, A^{\prime}\right)$ is less than one, then mapping 
$G$ is a contraction, consequently it has exactly one fixed point. Moreover, if the spectral radius of a matrix is less than one, then there exists a matrix norm, such that norm of the matrix is less then one, i.e. if $\rho\left(\frac{\lambda}{4} W^{*}\right)<1$, then there exist a matrix norm, such that $\left\|\frac{\lambda}{4} W^{*}\right\|<1$. Applying this matrix norm, mapping $G$ is a contraction, which completes the proof.

Since $\rho\left(W^{*}\right) \leq\left\|W^{*}\right\|$ for any matrix norm, Theorem 6 gives the best condition expressed by $W^{*}$.

Remark 1. The results in Sect. 4: Theorem 3, Theorem 4, Theorem 5 and Theorem 6 are valid for fuzzy grey cognitive maps with hyperbolic tangent threshold function $(\tanh (\lambda x))$, too, but we have to replace $4 / \lambda$ by $1 / \lambda$, since the derivative of $\tanh (\lambda x)$ is bounded by $\lambda$ (and not $\lambda / 4$ ).

\section{Example}

Let us consider the following weight matrix with imprecise (grey) entries:

$$
\otimes W=\left[\begin{array}{ccccccc}
0 & {[0.1,0.2]} & {[-0.6,-0.5]} & 0 & 0 & 0 \\
0 & 0 & {[-0.7,-0.5]} & 0 & 0 & {[0.1,0.3]} \\
{[0.6,0.8]} & 0 & 0 & {[-0.6,-0.2]} & 0 & 0 \\
{[0.7,0.9]} & 0 & 0 & 0 & {[0.6,0.8]} & 0 \\
0 & 0 & {[0.6,0.7]} & 0 & 0 & 0 \\
0 & {[0.1,0.3]} & 0 & {[0.8,1]} & {[-1,-0.8]} & 0
\end{array}\right]
$$

Then the matrix $W^{*}$ with the $w_{i j}^{*}$ entries:

$$
W^{*}=\left[\begin{array}{cccccc}
0 & 0.2 & 0.6 & 0 & 0 & 0 \\
0 & 0 & 0.7 & 0 & 0 & 0.3 \\
0.8 & 0 & 0 & 0.6 & 0 & 0 \\
0.9 & 0 & 0 & 0 & 0.8 & 0 \\
0 & 0 & 0.7 & 0 & 0 & 0 \\
0 & 0.3 & 0 & 1 & 1 & 0
\end{array}\right]
$$

The corresponding measures:

- Maximal weighted in-degree: 2.3

- Maximal weighted out-degree: 2

- Connectivity

- without self-feedback: $C o n_{w}=0.2633$

- with self-feedback: $C_{0} n_{w}=0.2194$

- spectral radius: $\rho\left(W^{*}\right)=1.1485$

According to Theorem 6, if $\lambda<3.4827$, then this grey FCM has one and only one grey fixed point. It also means that in this case the FGCM produces globally asymptotically stable behaviour, since every initial grey vector leads to the same equilibrium state. 


\section{Summary}

Fuzzy Grey Cognitive Maps are generalizations of classical FCMs, that can model the uncertainties of activation values and weights of causal connections.

In this paper, we provided some conditions for the convergence of FGCMs to a unique fixed point. The unicity of this attracting fixed point also ensures that the FGCM is globally exponentially stable, i.e. it converges to the same fixed point attractor regardless of the initial concept vector. Future work is focused on the effective detection of multiple fixed points scenarios and the prediction of oscillating patterns without simulations. The future goal is to provide exact analytical conditions for both of these behaviours.

Acknowledgment. The research presented in this paper was carried out as part of the EFOP-3.6.2-16-2017-00016 project in the framework of the New Széchenyi Plan. The completion of this project is funded by the European Union and co-financed by the European Social Fund.

This research was supported in part by National Research, Development and Innovation Office (NKFIH) K124055.

\section{References}

1. Harmati, I.Á., Kóczy, L.T.: On the convergence of sigmoidal fuzzy grey cognitive maps. Int. J. Appl. Math. Comput. Sci. 29(3), 453-466 (2019)

2. Harmati, I.Á., Hatwágner, M.F., Kóczy, L.T.: On the existence and uniqueness of fixed points of fuzzy cognitive maps. In: Medina, J., et al. (eds.) IPMU 2018. CCIS, vol. 853, pp. 490-500. Springer, Cham (2018). https://doi.org/10.1007/978-3-31991473-2_42

3. Harmati, I.A., Kóczy, L.T.: Notes on the dynamics of hyperbolic tangent fuzzy cognitive maps. In: 2019 IEEE International Conference on Fuzzy Systems (FUZZIEEE), New Orleans, LA, USA, pp. 1-6 (2019)

4. Liu, S., Lin, Y.: Grey Information. Springer, London (2006). https://doi.org/10. 1007/1-84628-342-6

5. Nápoles, G., Papageorgiou, E., Bello, R., Vanhoof, K.: On the convergence of sigmoid fuzzy cognitive maps. Inf. Sci. 349-350, 154-171 (2016)

6. Papageorgiou, E.I., Salmeron, J.L.: Methods and algorithms for fuzzy cognitive map-based decision support. In: Papageorgiou, E.I. (ed.) Fuzzy Cognitive Maps for Applied Sciences and Engineering (2013)

7. Rudin, W: Principles of Mathematical Analysis. McGraw-Hill Inc. (1964)

8. Salmeron, J.L.: Modelling grey uncertainty with fuzzy grey cognitive maps. Expert Syst. Appl. 37(12), 7581-7588 (2010)

9. Salmeron, J.L., Papageorgiou, E.I.: A fuzzy grey cognitive maps-based decision support system for radiotherapy treatment planning. Knowl.-Based Syst. 30, 151$160(2012)$

10. Salmeron, J.L., Palos-Sanchez, P.R.: Uncertainty propagation in fuzzy grey cognitive maps with Hebbian-like learning algorithms. IEEE Trans. Cybern. 49(1), 211-220 (2017)

11. Stylios, C.D., Groumpos, P.P.: Modeling complex systems using fuzzy cognitive maps. IEEE Trans. Syst. Man Cybern.-Part A Syst. Hum. 34(1), 155-162 (2004) 
12. Tsadiras, A.K.: Comparing the inference capabilities of binary, trivalent and sigmoid fuzzy cognitive maps. Inf. Sci. 178(20), 3880-3894 (2008)

13. Shojaei, P., Haeri, S.A.S.: Development of supply chain risk management approaches for construction projects: a grounded theory approach. Comput. Ind. Eng. 128, 837-850 (2019)

14. Boutalis, Y., Kottas, T.L., Christodoulou, M.: Adaptive estimation of fuzzy cognitive maps with proven stability and parameter convergence. IEEE Trans. Fuzzy Syst. 17(4), 874-889 (2009)

15. Knight, C.J., Lloyd, D.J., Penn, A.S.: Linear and sigmoidal fuzzy cognitive maps: an analysis of fixed points. Appl. Soft Comput. 15, 193-202 (2014) 